\title{
Mechanistic Model of Radiation-Induced Bystander Effects to Cells using Structured Population Approach
}

\author{
Fuaada Mohd Siam* and Muhamad Hanis Nasir \\ Department of Mathematical Sciences, Faculty of Science \\ Universiti Teknologi Malaysia, 81310 Johor Bahru, Johor, Malaysia \\ *Corresponding author: fuaada@utm.my
}

Article history

Received: 21 September 2018

Received in revised form: 4 December 2018

Accepted: 17 December 2018

Published on line: 31 December 2018

\begin{abstract}
In irradiation process, instead of traverse on the targeted cells, there is side effect happens to non-targeted cells. The targeted cells that had been irradiated with ionizing radiation emits damaging signal molecules to the surrounding and then, damage the bystander cells. The type of damage considered in this work is the number of double-strand breaks (DSBs) of deoxyribonucleic acid (DNA) in cell's nucleus. By using mathematical approach, a mechanistic model that can describe this phenomenon is developed based on a structured population approach. Then, the accuracy of the model is validated by its ability to match the experimental data. The Particle Swarm (PS) optimization is employed for the data fitting procedure. PS optimization searches the parameter value that minimize the errors between the model simulation data and experimental data. It is obtained that the mathematical modelling proposed in this paper is strongly in line with the experimental data.
\end{abstract}

Keywords Bystander effects; double-strand breaks; particle swarm optimization; cell survival fraction; parameter estimation.

Mathematics Subject Classification 92B99, 37N25, 37N40.

\section{Introduction}

Mathematical modelling for real life phenomenon requires the knowledge of science of the biological phenomenon and the corresponding real or experimental data. Biological phenomenon is a complex process and full of mysteries. It offers great opportunities to those who are seeking the knowledge of biological phenomenon and hence improving the quality of life for humanity [1]. In this paper, a mathematical analysis on the effects of ionizing radiation towards bystander cells is presented. A related experimental data taken from the literature is used in performing the model validation.

Nowadays, millions of people around the world undergo radiotherapy [2]. Radiotherapy is one of the cancer treatment alongside with other types of treatments such as surgery, chemotherapy, immunotherapy, hormone therapy and etc. In Malaysia, Zahrina et al. [3] stated that the 
chances of Malaysian people to get cancer illness is in ratio 1:4 peoples, which is a quite high probability. Radiotherapy treatment uses high dose energy of ionizing radiation to destroy the unwanted cells, such as cancer cells. DNA damage will occur when the ionizing radiation hit the targeted cell. It will cause the cell-cycle not be able to proceed and the apoptosis happened. However, the irradiation also can affect cell surrounding targeted cells (bystander cells) and caused DNA lesions. Ionizing radiation enhance the frequency of secondary effects of cells located in the vicinity of the irradiated cells, which is widely known as radiation-induced bystander effects (RIBE) [4]. The irradiated cells refer to cells that have been traversed by ionizing radiation. RIBE is a phenomenon resulting from danger signal molecules (bystander signals) emitted by the irradiated cells (see Figure 1). The affected bystander cells show many biological effects such as single-strand breaks, double-strand breaks (DSBs), chromosomal aberrations, sister chromatid exchange, carcinogenesis, micronucleation, increased frequency of apoptosis, reduced clonogenic efficiency and oncogenic transformation and etc $[5,6,7,8,9]$.

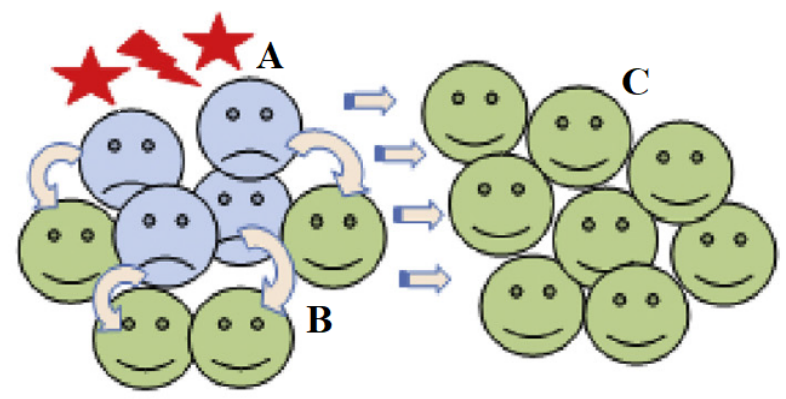

Figure 1: A Cartoon Representation of Targeted Cells Releases Some Signals to Bystander Cells [10]

Verma and Tiku [10] proposed the schematic representation of bystander effects phenomenon (see Figure 1). Cells A are the cells that traversed directly by irradiation, cells B are the adjacent cells and cells $\mathrm{C}$ are the distant cells or tissue. After cells A had been traversed by irradiation, an oxidative DNA lesion is formed and cells A become the irradiated cells. According to Han and $\mathrm{Yu}[11$, bystander signal molecules are produced very quickly which is less than 2.5 minutes from irradiated cells after irradiation process. Bystander response happens in cells B because of signaling process via gap junction intercellular communication, while cells $\mathrm{C}$ becomes affected because of signaling process via bystander factors released into the cultured medium, that is distant cell signaling intercellular communication [12].

The bystander signal molecules trigger negative feedback to unirradiated cells by causing DNA damage and DNA repair delay [13]. There are many kinds of bystander signal molecules produced by the irradiated cells. The molecule of bystander signals may consist of reactive oxygen species, interleukin-1, reactive nitrogen species, interleukin-6, transforming growth factor $\beta-1$, interleukin-8, nitric oxide, tumor necrosis factor- $\alpha$ and etc $[6,14,15,16]$. There are many experimental evidence has been done on this phenomenon, interested reader may refer to Table 1 in Hatzi et al. [17]) and Table 1 in Widel [15]) for the summary of experimental works related to RIBE in vivo and in vitro, respectively.

In this work, the damage considered is the number of DSBs on DNA inside cell's nucleus. The DSBs are considered as the insidious lesions of DNA damage and also considered as the 
simplest form representing the complex DNA damage $[6,16,18]$. The interaction of two DSBs can result in chromosome aberrations, mutation, carcinogenesis and cell death [19]. There are many types of repair mechanisms, such as DNA inter-strand cross link repair, mismatch repair, direct damage reversal, nucleotide excision repair, base excision repair, non-homologous end joining and homologous recombination repair [20]. However, some of the damages are not repaired or are mis-repaired [21].

Some mathematical modelling frameworks on the irradiation bystander effects phenomenon have been discussed in previous studies. In 2009, Xia et al. [22] developed a Monte-Carlo model in order to study the bystander effects mechanism under the cells sparsely population conditions. Same as Sasaki et al. [23], they also used a Monte-Carlo technique to study the diffusion of soluble factors in bystander response. In 2012, Kundrát and Friedland [24] studied about the non-linear response of cells to bystander signals, which it is interpreted by a sigmoid response function. In 2014, Lintott et al. [25] presented a Bio-PEPA model that used a process algebra method, which the model combined the epidemic-type structure with biological mechanism of bystander effects. In 2015, Hattori et al. [26] developed a simulation system that is based on a two-dimensional cellular automaton. There are four components in the simulation system, which is the irradiation of cells, generation and diffusion of bystander signals, induction of cellular DNA damage and cellular response by DNA damage. In 2017, Olobatuyi et al. [13] developed a reaction-diffusion model to investigate the dynamics of the lifespan of a bystander signal emitted by the irradiated cells. In continuation of studying the bystander effects phenomenon, a new mathematical modelling framework is introduced based on a structured cell population approach.

In 2018, the structured cells population model developed by Siam et al. [27] used the idea of total number of double-strand breaks (DSBs) in cell's nucleus as the corresponding characteristic of DNA damage. Siam et al. [27] developed the model in order to represent the Linear-Quadratic (LQ) relation. LQ relation is commonly used to explain the relationship between cell surviving fraction and radiation dose to the phenomenon of irradiation effects on targeted cells. There is evidence showed that the mechanism between targeted and non-targeted effect on the DSBs on the DNA is the same [13, 28], thus in this research, the Siam et al. [27] model is employed to explain the biological process of the RIBE phenomenon. The nature of analysis used in this paper is forward and inverse modelling. A forward modelling is a detailed mathematical model set up for the phenomenon investigated, while inverse modelling is a data fitting procedure that uses optimization algorithm in finding the best fit between simulation data and experimental data [29].

The experimental data used in this work is taken from Boyd et al. [30]. They performed an experiment on human tumor cell line UVW (glioma) in order to compare the induction of bystander effects by external beam $\gamma$-radiation with exposure to three radiohaloanologs of metaiodobenzylguanidine (see Figure 2). The experiment was carried out by using medium transfer method. Medium from cells that were treated with $\gamma$-radiation was transferred to cells that had not been exposed to irradiation. Then, clonogenic survival was determined in targeted and bystander cells. The result shows that over the dose range 0-9 Gy, there are 30\% to $40 \%$ of clonogenic cell killing in bystander cells but this potency was maintained at higher dosage. In the inverse modelling, the experimental data of bystander cells in Figure 2 is used for estimating the value of parameters in the model. 


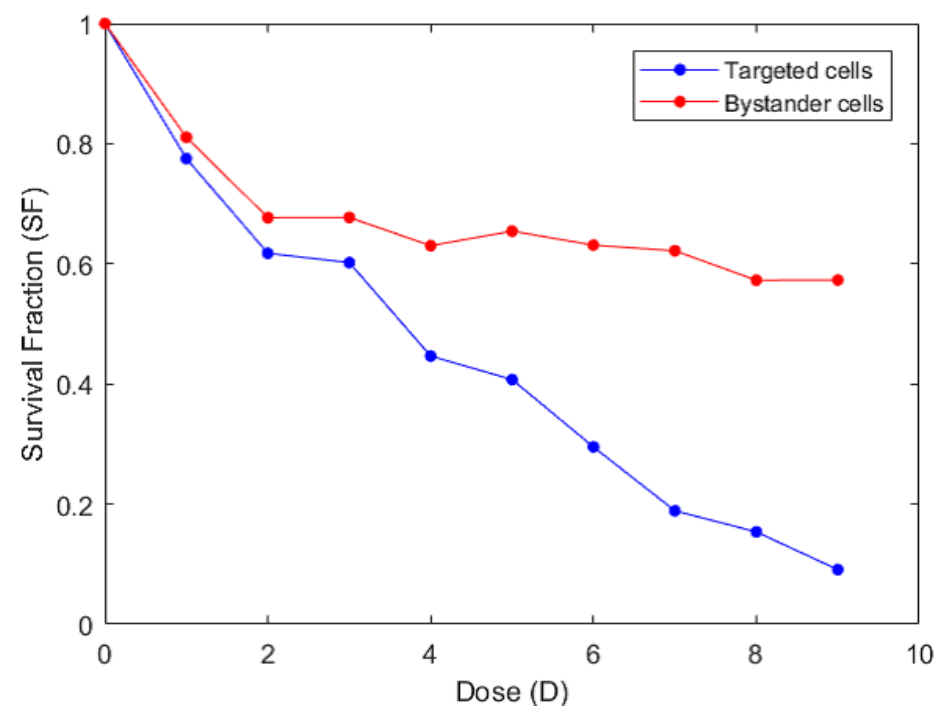

Figure 2: Experimental Data from Boyd et al. [30] for Targeted and Bystander Cells

\section{Methodology}

\subsection{Modelling Formulation}

The bystander cells react with bystander signals emitted by the irradiated cells and behave as if they have been directly affected by ionizing radiation [13, 28]. According to the experimental procedure done by Yang et al. [31] using the insert dish co-culture system (see Figure 3(a)), there is an increase in the percentage of $\gamma$-H2AX positive cells was detected in the non-targeted cell population at 2 hours after co-culturing with X-ray irradiated cells immediately after irradiation. The $\gamma$-H2AX foci is a phosphorylated form of histone H2AX that acted as a biomarker of DSBs formation $[6,11,16,32]$.

(a)

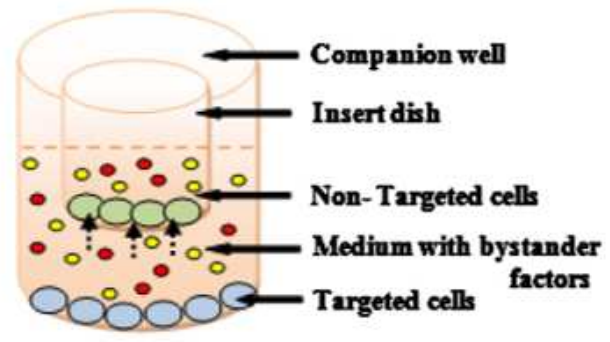

(b)

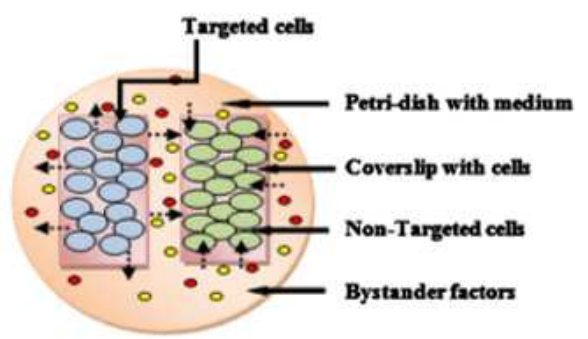

Figure 3: Methods used to Study Bystander Response Experimentally [10] 
The mathematical modelling framework in this work is assumed follows the experimental method shown in Figure 3(b). The targeted cells were cultured on the left coverslip and the bystander cells were cultured on the right coverslip. In addition, the petri-dish contains medium that allows bystander signals (bystander factors) to move freely and interact with bystander cells. This work brings up an idea of 10000-targeted cells had been irradiated with a specific radiation dose and the survival of 10000-bystander cells were observed after a time interval.

There are three assumptions made in the modelling framework. First, all bystander cells receive the same amount (or strength) of bystander signals. At certain dose (D) value, the bystander signals produced follow Equation (11). Second, the bystander signals produced by irradiated cells caused DNA damage in bystander cells that is in the form of molecular bond breaks, double-strand breaks (DSBs). Third, the repair mechanism in bystander cells experience a repair delay effects and DSBs can be repaired and mis-repaired. There are also two limitations that are not included in this modelling. First, repopulation and cell cycle is not included in this model. Cell cycle phases play an important role in cell population because in the cell division phase, cells are divided into two [18]. Second, the model assumes that the cells continuing the process of repairing and death without any resting phase because in certain biological condition, the cell can enter a quiescence state [33]. It is known that the process of intercellular and intracellular in radiation induced bystander effects is a complex process. In this modelling, there are only DSBs repair mis-repair and cells death.

\subsubsection{Model of Repair Mis-repair DNA DSBs Damage}

Based on the assumptions stated in previous section, the DSBs are formed in bystander cells after react with bystander signals. Therefore, the model of repair and mis-repair DNA DSBs damage developed by Siam et al. [27] is employed. The variable $N_{k, m}$ refers to a group of cells that have $k$ DSBs repair and $m$ mis-repair DSBs. The model is as follows:

$$
\frac{d N_{k, m}}{d t}=-\beta(k, m) N_{k, m}-\gamma(k) N_{k, m}+p \gamma(k+1) N_{k+1, m}+(1-p) \gamma(k+1) N_{k+1, m-1},
$$

for $k=0,1,2, \ldots, k_{\max }, m=0,1,2, \ldots, k_{\max }$ with a pairing condition of $k$ and $m$ such as $k+m \leq k_{\max }$ and $k_{\max }$ is the maximum number of DSBs in a population of bystander cells. The parameter $\beta, \gamma$ and $p$ are the death rate, the repair rate and the probability of successful repair 1 DSB, respectively.

The death rate function, $\beta$ is considered in two ways, that is due to mis-repair of a DSB and interaction of two DSBs located in spatial proximity formed a lethal chromosomal aberration. Hence, the death rate is presented as:

$$
\beta(k, m)=\alpha_{1} m+\alpha_{2} k^{2},
$$

where $\alpha_{1}$ is a mis-repair rate constant while $\alpha_{2}$ is a lethal damage rate constant.

The repair rate function, $\gamma$ is the rate of DSB repair for cells having $k$ DSBs. The repair process is described as follows:

$$
\gamma(k)=\frac{V_{\max } k}{K_{M}+k}
$$


where $k=0,1,2, \ldots$ is the number of DSBs, $V_{\max }$ is the maximum repair rate and $K_{M}$ is a constant at which $V_{\max }$ is halved.

Another critical effect of bystander effect phenomenon is DNA repair delays [13, 34]. In simulation obtained by Powathil et al. [34], the normal cells that are responding to the bystander signals are assumed to undergo repair delay up to 6 hours. The repair delay effect is captured by using a Heaviside step function. The Heaviside step function is defined as follows:

$$
h(t)=\left\{\begin{array}{lll}
0 & \text { if } \quad t \leq \tau \\
1 & \text { if } \quad t>\tau
\end{array}\right.
$$

where $\tau$ is the time of delay before repair process begin. Therefore, the repair rate with DNA repair delay can be rewritten as:

$$
\gamma(k, h)=\frac{V_{\max } k h}{K_{M}+k}
$$

The repair rate function will be activated depends on the duration of delay.

Based on Equation (5), it is suggested that the model of repair-mis-repair DNA DSBs damage with delay activation of repair process is written as:

$$
\frac{d N_{k, m}}{d t}=-\beta(k, m) N_{k, m}-\gamma(k, h) N_{k, m}+p \gamma(k+1, h) N_{k+1, m}+(1-p) \gamma(k+1, h) N_{k+1, m-1}
$$

\subsubsection{Distribution of Initial Condition using Poisson Random}

For the initial condition, the DNA DSBs damage is formed in the bystander cells by considering $\gamma$-H2AX foci formation, a direct biomarker of DSBs. Assumption made by Hattori et al. [26] stated that the number of DSBs formed after being in contact with bystander signals is proportional to the quantities of bystander signals. Therefore, the probability of a bystander cell acquiring $k$ DSBs follows the Poisson distribution with average:

$$
\mu=\vartheta C
$$

where $\vartheta$ is the DSBs induction coefficient among the bystander cells and $C$ is the bystander signals. The model of bystander signals used is taken from Kundrát and Friedland [24]. The model is as follows:

$$
C=1-\exp \left\{-\mathrm{D} / D_{c}\right\}
$$

where $C$ is the relative signal concentration emitted into the medium by the irradiated cells with dose $(\mathrm{D})$ and $D_{C}$ is the value of characteristic sensitivity of the irradiated cells. Note that the diffusion of signal is not considered here, only the final steady-state concentration of signal in medium are considered which are likely reached within 30 minutes and the signals remain steady for at least 6 hours after radiation [24, 34].

By using Poisson distribution function, the probability of one cell acquiring $k \geq 0$ DSBs after being in contact with signals is given by:

$$
P(\mathrm{DSBs}=k)=\frac{\mu^{k} e^{-\mu}}{k !} .
$$

The initial distribution only generate the number of DSBs $k$, therefore in the initial condition, set $N_{k, m}(0)=0$ for $m \geq 1$, for all $k$. 


\subsubsection{General Solution of the Model and Example of Initial Condition $\mathrm{N}_{0}$ and Matrix A}

The model of Equation (6) takes form of derivative,

$$
\frac{d \mathbf{N}}{d t}=\mathbf{A} \mathbf{N}
$$

which depends on the value of $k_{\max }$ in a population of bystander cells. The number of ODEs exist in vector $\mathbf{N}$ is $M$ and dimension of matrix $\mathbf{A}$ is $M \times M$ with:

$$
M=\frac{\left(k_{\max }+1\right)\left(k_{\max }+2\right)}{2} .
$$

According to [35], the solution for this initial value problem is as follows:

$$
\mathrm{N}(t)=e^{\mathbf{A}\left(t-t_{0}\right)} \mathbf{N}_{0}
$$

where $\mathbf{N}_{0}$ is the vector of initial condition at initial time, $t_{0}$. Reader can refer to [36] on how to compute the survival fraction of bystander cells.

For example, suppose there are 20 bystander cells affected by bystander signals. The discrete number of DSBs will be generated by Equation (9) implemented in "poissrnd" MATLAB function. Suppose the number of DSBs in each bystander cell are 1,0,0,1,1,1,0,0,1,0,1,1,0,1,0,1,1,0,0 and 1, respectively. Observed that the biggest number of DSBs is $1, k_{\max }=1$. By Equation (11), the value of $k_{\max }=1$ create $M=3$ groups of bystander cells, which is $\mathbf{N}=\left\{N_{0,0}, N_{1,0}\right.$ and $\left.N_{0,1}\right\}$. Then, the initial condition will be the total of bystander cells according to number of DSBs. The initial condition, $\mathbf{N}_{\mathbf{o}}$ for this example is as follows:

$$
\left[\begin{array}{l}
N_{0,0}(0) \\
N_{1,0}(0) \\
N_{0,1}(0)
\end{array}\right]=\left[\begin{array}{c}
9 \\
11 \\
0
\end{array}\right] .
$$

For $k_{\max }=1$ and the vector $\mathbf{N}=\left\{N_{0,0}, N_{1,0}\right.$ and $\left.N_{0,1}\right\}$, the ODEs system based on Equation (6) is as follows:

$$
\begin{aligned}
& \frac{d N_{0,0}}{d t}=-\beta(0,0) N_{0,0}-\gamma(0, h) N_{0,0}+p \gamma(1, h) N_{1,0} \\
& \frac{d N_{1,0}}{d t}=-\beta(1,0) N_{1,0}-\gamma(1, h) N_{1,0} \\
& \frac{d N_{0,1}}{d t}=-\beta(0,1) N_{0,1}-\gamma(0, h) N_{0,0}+(1-p) \gamma(1, h) N_{1,0}
\end{aligned}
$$

The ODEs in Equation (14) can be rewritten into matrix, $\frac{d \mathbf{N}}{d t}=\mathbf{A N}$ as follows:

$$
\frac{d}{d t}\left[\begin{array}{l}
N_{0,0} \\
N_{1,0} \\
N_{0,1}
\end{array}\right]=\left[\begin{array}{ccc}
-\beta(0,0)-\gamma(0, h) & p \gamma(1, h) & 0 \\
0 & -\beta(1,0)-\gamma(1, h) & 0 \\
0 & (1-p) \gamma(1, h) & -\beta(0,1) N_{0,1}-\gamma(0, h)
\end{array}\right]\left[\begin{array}{c}
N_{0,0} \\
N_{1,0} \\
N_{0,1}
\end{array}\right]
$$

Therefore, by applying Equation (12) that is the general solution for this type of initial value problem, the solution for this example is as follows: 


$$
\left[\begin{array}{l}
N_{0,0}(t) \\
N_{1,0}(t) \\
N_{0,1}(t)
\end{array}\right]=\exp \left\{\left[\begin{array}{ccc}
-\beta(0,0)-\gamma(0, h) & p \gamma(1, h) & 0 \\
0 & -\beta(1,0)-\gamma(1, h) & 0 \\
0 & (1-p) \gamma(1, h) & -\beta(0,1) N_{0,1}-\gamma(0, h)
\end{array}\right] t\right\}\left[\begin{array}{c}
9 \\
11 \\
0
\end{array}\right] .
$$

\subsubsection{Steps of Simulation on Survival Fraction of Bystander Cells}

MATLAB R2017a is employed to perform the simulation of the mathematical model. The algorithm contains eight steps in order to compute the survival fraction (SF) of bystander cells with respect to dose (D). The steps of algorithm are as follows:

Step 1: Generate the random initial condition, $\mathbf{N}_{0}$ by using Poisson distribution function with average DSBs formation in bystander cells, $\mu=\vartheta C$ (see Equation (7)). The irradiation dose $(\mathrm{D})$ is incorporated into the system through signal concentration in Equation (8).

Step 2: Compute the model Equation (12) for up to $T=24$ hours. As suggested by [36], the complete one cell-cycle is approximately within 24 hours. Therefore, the simulation is limited into 24 hours as cell-cycle progression is not included in current model formulation.

Step 3: Since the DNA repair delay effect need to be considered, the system will be solved in two different situations. Firstly, compute the Equation (12) at delay time $\tau, \mathbf{N}(\tau)$ $=\exp \{\mathbf{A} \tau\} \mathbf{N}_{0}$. The repair rate is zero at this duration due to the Heaviside step function, Equation (4).

Step 4: Second, compute the Equation (12) for the next $t=T-\tau$ by changing $\mathbf{N}_{0}$ with $\mathbf{N}(\tau)$ obtained in Step 3, $\mathbf{N}(T)=\exp \{\mathbf{A}(T-\tau)\} \mathbf{N}_{0}$. Thus, the model is investigated in a duration of 24 hours.

Step 5: Compute the survival fraction of bystander cells, $\mathrm{SF}=\sum \mathbf{N}(T) / \sum \mathbf{N}_{0}$.

Step 6: The aim of the algorithm is to plot $\mathrm{SF}$ versus $\mathrm{D}$ since the available experimental data is in the form of SF versus D. Generate 41 simulation data of SF with the increasing value of dose D by 0.25 Gy.

Step 7: Due to the randomness of the initial condition, Step 1 to 6 is repeated for 10 times and then compute the average value of 41 simulation data of SF.

Step 8: Plot SF versus D.

\section{$2.2 \quad$ Data Fitting of the Model}

The goal of parameter fitting is to determine the parameter value for which model simulation best matches with the experimental data [29, 37]. The method used in this work is sum-squared error (SSE) minimization [38]. SSE is defined as follows:

$$
\mathrm{SSE}=\sum\left[y_{i}\left(x_{i}\right)-\hat{y}_{i}\left(x_{i}\right)\right]^{2},
$$


where $y_{i}$ is the experimental data, $y_{i}$ is the model simulation data and $i=1,2, \ldots, n$ is for $n$ available experimental data. In this paper, the SSE minimization is achieved by using optimization method. The Particle Swarm (PS) optimization is employed by considering SSE as the objective function. The PS optimization is explained briefly in Section 2.2.1.

There are eight parameters introduced in Section 2.1. The parameters in the model are $\vartheta$, $D_{C}, \alpha_{1}, \alpha_{2}, p, V_{\max }, K_{M}$ and $\tau$. The parameter boundary for all parameters are employed based on suggestion from the previous studies (see Table 1).

Table 1: Boundaries of All Parameters

\begin{tabular}{|c|c|c|c|}
\hline Parameter & Lower Boundary & Upper Boundary & Reference \\
\hline$\vartheta\left(C^{-1}\right)$ & 1.8 & 9.7 & {$[39]$} \\
\hline$D_{C}(\mathrm{~Gy})$ & 0.003 & 3 & {$[24]$} \\
\hline$\alpha_{1}\left(h^{-1}\right)$ & 0.0277 & 20.79 & {$[27]$} \\
\hline$\alpha_{2}\left(h^{-1}\right)$ & 0 & 0.005 & {$[27]$} \\
\hline$p$ & 0 & 1 & - \\
\hline$V_{\max }\left(h^{-1}\right)$ & 0.1 & 3 & {$[27]$} \\
\hline$K_{M}$ & 0 & 5 & {$[6,34]$} \\
\hline$\tau(h)$ & 0.05 & 6 & \\
\hline
\end{tabular}

Note: unit $\left(C^{-1}\right)$ is per relative signals concentration,

unit (Gy) is gray, unit $(h)$ is hour and unit $\left(h^{-1}\right)$ is per hour

According to the experimental work done by Belchior et al. [39], the result stated that the average double-strand breaks (DSBs) foci formation in bystander cells (data of different distance from the irradiated cells and different value of dose) are in the range of 1.8 to 9.7 foci. It can be suggested that the parameter $\vartheta \approx 1.8-9.7 C^{-1}$. Kundrát and Friedland [24] suggested that the distribution of characteristic doses for signal emission in the irradiated cell population assumed to be $70 \%$ cells emit signals with $D_{C}=0.003$ Gy, $10 \%$ with $D_{C}=0.03$ Gy, $10 \%$ with $D_{C}=0.3$ Gy and $10 \%$ with $D_{C}=3$ Gy. Thus, $D_{C}$ can be suggested in the range of $D_{C} \approx 0.003-3$ Gy. For parameter $\alpha_{1}$ and $\alpha_{2}$, Siam et al. [27] reported the result from survival data of $\mathrm{CHO}$ cells and pointed out that $\alpha_{1} \approx 0.0277-20.79 h^{-1}$ and $\alpha_{2} \approx 0$ - $0.005 h^{-1}$. For parameter $V_{\max }$ and $K_{M}$, Siam et al. [27] reported $V_{\max } \approx 0.1-3 h^{-1}$ and $K_{M} \approx 0-5$, where it is obtained from the results of 11 experimental survival data sets with different cell line and different types of irradiation. Wang et al. [6] reported that the repair kinetics (ataxia-telangiectasia mutated foci) could be observed in bystander cells after $0.05 h$ and Powathil et al. [34] suggested that the bystander cells could undergo repair delay for up to $6 \mathrm{~h}$. Therefore, $\tau$ can be suggested in range of $\tau \approx 0.05-6 h$. 


\subsubsection{Particle Swarm (PS) Optimization}

The theory of PS optimization was developed from swarm intelligence and its inspired upon the random behavior of bird flocking in the air or fish schooling in water [40]. PS optimization uses a number of particle vectors moving around in the space searching for the optimal solution. A swarm of $B$-particle is $\left\{\mathbf{x}_{1}, \mathbf{x}_{2}, \ldots, \mathbf{x}_{B}\right\}$. Each particle, $\mathbf{x}_{i}$ acts as a point of $D$-dimensional space, i.e.: $x_{i, j}=\left[x_{i, 1}, x_{i, 2}, \ldots, x_{i, D}\right]$ for $i=1,2, \ldots, B$. Every particle keeps the information in the solution space in all iterations, i.e.: $\mathbf{x}_{i}^{k}$ for $k=1,2, \ldots, K$ (maximum number of iteration).

The best solution by each particle is called personal best, $\mathbf{P}_{i}^{k}$ for $i=1,2, \ldots, B$ and up to iteration $k$. This solution is obtained according to the personal experiences of each particle vector. The best value that will be tracked in the population of particles is called global best, $\mathbf{G}^{k}$ for up to iteration $k$. The particle vectors which move freely into the space is in the form of swarm and the velocity vectors and position of each particle is updated according to the value of $\mathbf{P}_{i}^{k}$ and $\mathbf{G}^{k}$.

Each particle is followed by its coordinates in the search space for optimization of $\mathbf{G}^{k}$ solution. The particle velocity continuously adjusted according to the particle's position. The velocity of a particle $\mathbf{x}_{i}$ is given as $\mathbf{v}_{i}=\left[v_{i, 1}, v_{i, 2}, \ldots, v_{i, D}\right]$ for $i=1,2, \ldots, B$. The initial velocity of the swarm is denoted as $\mathbf{v}=\left[\mathbf{v}_{1}, \mathbf{v}_{2}, \ldots, \mathbf{v}_{B}\right]$. As reviewed in [41], the equation for velocity is as follows:

$$
v_{i, j}^{k+1}=\omega^{k} v_{i, j}^{k}+c_{1} \gamma_{1}\left(p_{i, j}^{k}-x_{i, j}^{k}\right)+c_{2} \gamma_{2}\left(G_{j}^{k}-x_{i, j}^{k}\right),
$$

where $\omega^{k}$ is the particle's inertia function, $c_{1}$ and $c_{2}$ are the acceleration constants and $\gamma_{1}$ and $\gamma_{2}$ are the uniformly distributed random value generated between 0 and 1 . Then, the position of each vector is updated by:

$$
x_{i, j}^{k+1}=x_{i, j}^{k}+v_{i, j}^{k+1} .
$$

According to $[42,43]$, the necessary and sufficient condition for stability of the swarm are as follows:

$$
c_{1}+c_{2}<4 \text { and } \frac{c_{1}+c_{2}}{2}-1<\omega<1
$$

These conditions can guarantee the convergence to a stable equilibrium. The parameter $c_{1}$ and $c_{2}$ also known as cognitive attraction and social attraction, respectively. The particle's inertia function, $\omega^{k}$ is defined as:

$$
\omega^{k}=0.9-\frac{0.4(k-1)}{K-1} .
$$

A linearly decreasing particle's inertia function from around 0.9 to around 0.4 during exploration in the search space [43].

The graphical representation on how a particular particle, $\mathbf{x}_{i}$ moving around in the search space is shown in Figure 4. The movement of new position $\mathbf{x}_{i}^{k+1}$ is always move to attraction towards $\mathbf{P}_{i}^{k}$ and $\mathbf{G}^{k}$. Figure 4 is for the case of $D=2$ since 2-dimensional space is easy to visualize, i.e.: $\mathbf{x}_{i}=\left\{x_{i, 1}, x_{i, 2}\right\}$. In this paper, the analysis using PS optimization is implemented by using a built-in MATLAB function called "particleswarm" and the default parameters in the PS optimization is set as $D=8, c_{1}=0.5, c_{2}=1.25, K=150$ and $B=10$. 


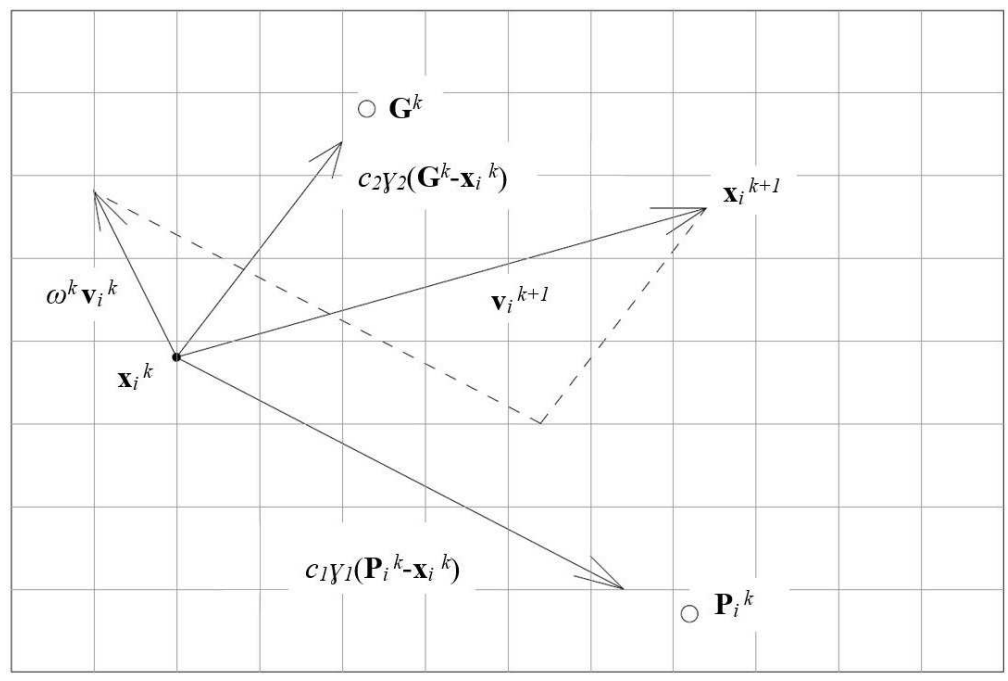

Figure 4: The 2-dimensional PS Optimization [42]

\section{Results and Analysis}

\subsection{Parameter Estimation of the Model}

In this work, 50 sets of parameters value have been estimated. Note that different set of initial point give different result of parameters value estimated but the result of finding the minimum SSE between model simulation data and experimental data is successfully achieved. Thus, 50 different sets of initial points are chosen in the range suggested in Table 1 and the results of 50 sets of parameters estimated value are analyzed statistically (see supplementary file A for details). The value of sample mean and 95\% confidence level are calculated in order to investigate the variation of 50 sets of estimated parameter.

Table 2: Summary of Parameter Estimation Results

\begin{tabular}{|c|c|c|}
\hline Parameter & Mean & 95\% C.I. \\
\hline$\vartheta$ & 3.8001 & {$[3.4370,4.1631]$} \\
\hline$D_{C}$ & 1.6694 & {$[1.6116,1.7272]$} \\
\hline$\alpha_{1}$ & 10.0548 & {$[8.5792,11.5303]$} \\
\hline$\alpha_{2}$ & 0.0014 & {$[0.0011,0.0018]$} \\
\hline$p$ & 0.8614 & {$[0.8401,0.8828]$} \\
\hline$V_{\max }$ & 1.9514 & {$[1.7181,2.1846]$} \\
\hline$K_{M}$ & 2.5126 & {$[2.1926,2.8326]$} \\
\hline$\tau$ & 2.0053 & {$[1.5671,2.4436]$} \\
\hline
\end{tabular}




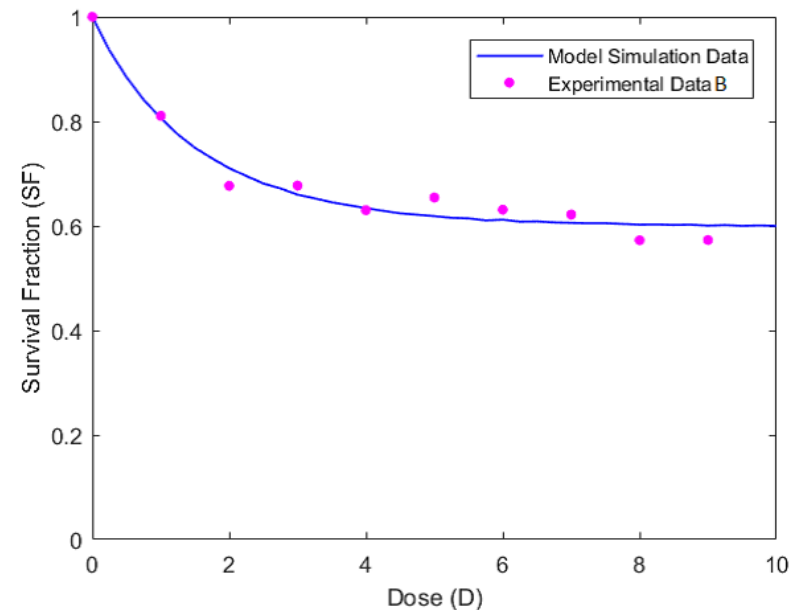

(a)

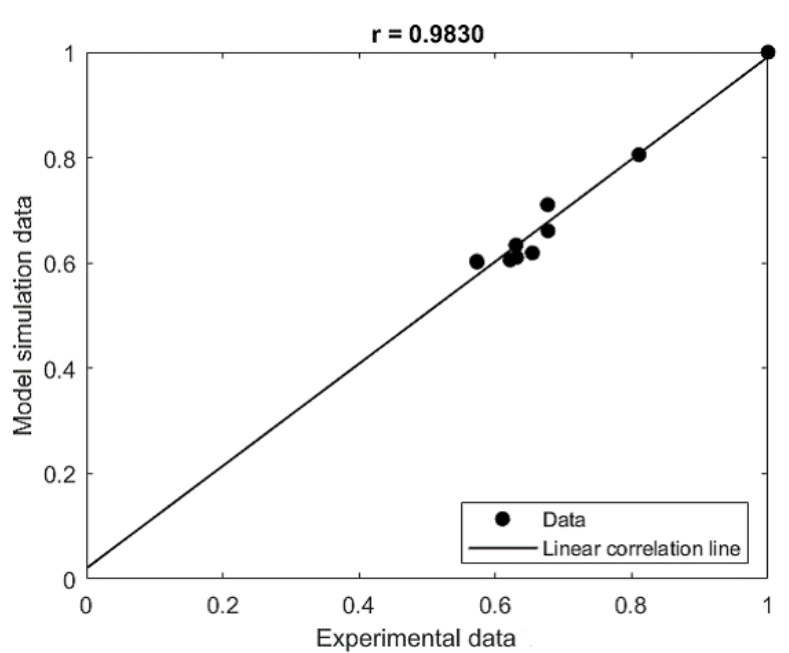

(b)

Figure 5: (a) Illustration of Model using One Set of the Estimated Parameter; (b) The Model Simulation Data versus the Experimental Data

Using PS optimization, the mean value of $\mathrm{SSE}=0.0053$ is reasonably small value; this indicates a good agreement between model simulation data and experimental data. Efficiency of PS optimization then verified by determining the correlation, $r$ between the experimental data and model simulation data obtained from estimated parameters value. The result shows the mean value of the correlation, $r=0.9825$ between estimated model simulation data and experimental data is close to 1 which corresponding to an excellent fit by PS optimization. As shown in Table 2, the mean of all parameters is within the interval with $95 \%$ confident level. Therefore, the estimated parameter shall be applied again in the model in order to provide a graphical plot of model simulation versus experimental data.

As an illustration, Figure 5(a) shows the simulation of the model by using one set of parameter value from 50 samples set of estimated parameter. The estimated parameters are $\vartheta=$ $2.2072 C^{-1}, D_{C}=1.7959$ Gy, $\alpha_{1}=7.2906 h^{-1}, \alpha_{2}=0.0020 h^{-1}, p=0.7740, V_{\max }=2.1472 h^{-1}$, $K_{M}=1.9953$ and $\tau=0.0510 h$ (set no. 49 in supplementary file A). The corresponding value of SSE and $r$ is 0.0051 and 0.9830 , respectively. The linear correlation plot between simulation and experimental data also shown in Figure 5(b).

\subsection{Discussion}

It is important to analyze the overall effects of irradiation to cells. The intention of ionizing radiation is to give damage as much as possible to the unwanted cells and avoid damaging the non-targeted cells. The bystander effects are a complicated phenomenon and yet not fully understood. Hundreds of scientific articles that are related to the bystander effects phenomenon have been published [8]. In order to analyze the survival of bystander cells, a mathematical model that based on structured cells population is developed in this paper. In the field of experimental studies, many quantitative data have been published. Thus, model suggested can be validated by its ability and accuracy to reproduce the experimental data.

The choice of parameter values for model is important for matching the experimental data 
and making predictions from the model. Parameter fitting is an essentially an optimization procedure that will search the parameter of model and minimizing sum-squared error between model simulation data and experimental data [44]. Parameter fitting is become easier nowadays due to the development of optimization packages in MATLAB R2018a. As for PS optimization used in the parameter fitting procedure, the MATLAB built-in function is called "particleswarm". There are many other types of optimization built-in function have been developed in MATLAB. These functions are able to fit a variety of experimental data and suitable for any type of mathematical models.

From Table 2, value of parameter $D_{C}=1.6694$ Gy is the characteristic sensitivity of the targeted cells. This value is used in Equation (11) for the bystander signals model. The relative bystander signals depend on the radiation dose and the signal secretion is normalized to unity at higher doses [24]. As shown Figure 6, the signal concentration increase as higher dose given to the targeted cells. The bystander signals secretion can be suggested to follow a saturating Michaelis-Menten equation, so that possible maximum signal concentration released could be analyzed. Various type of bystander signals contributes to the bystander response. The incubation of bystander cells with superoxide dismutase (a superoxide radical scavenger) able to inhibit the bystander effects such as sister chromatid exchanges [16].

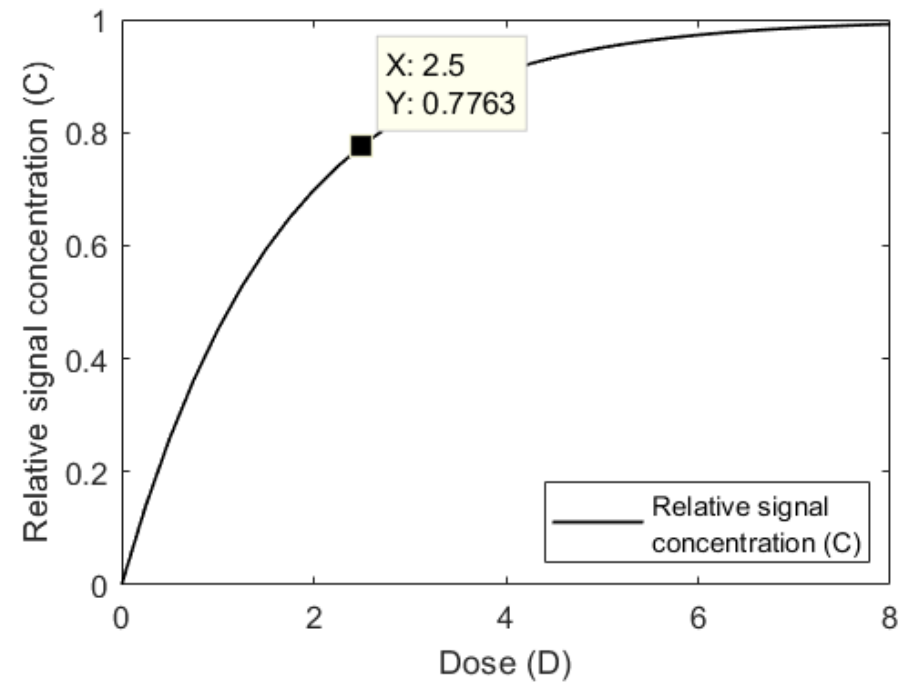

Figure 6: Relative Signal Concentration using $D_{C}=1.6694$ Gy

The biological effects in bystander cells affected by bystander signals is numerous. In this modelling, the damage considered is DNA DSBs damage. The number of DSBs damage experience by the bystander cells are assumed to follow Poisson distribution function. The MATLAB syntax for generating Poisson random number is "poissrnd". From Table 2, $\vartheta=3.8001 C^{-1}$ and in Figure 6, the value of relative signal concentration, $C=0.7763$ at dose $\mathrm{D}=2.5 \mathrm{~Gy}$. Thus at D $=2.5 \mathrm{~Gy}$, the average number of DSBs induced in bystander cells population is $\mu$ $=2.9500$ DSBs. DSBs are lethal to bystander cells, as they can form lethal chromosomal aberrations. Two DSBs can caused intrachromosomal paracentric inversions, interstitial deletions, pericentric inversions and interchromosomal translocations [45].

The death rate function depends on number of DSBs $(k)$ and mis-repair DSBs $(m)$. From Table 2, $\alpha_{1}=10.0548 h^{-1}$ and $\alpha_{2}=0.0014 h^{-1}$. The death rate will be different in each group 
of cells, such as the death rate for group $N_{2,1}$ will be $\beta(2,1)=10.0604 h^{-1}$ and for group $N_{5,1}$ will be $\beta(5,1)=10.0898 h^{-1}$. The group of bystander cells that has many DSBs tends to have more possibility of lethal chromosomal aberrations. There are two dominant DNA repair pathways that deal with DSBs lesion, which is non-homologous end-joining and homologous recombination [46]. In Table 2 , the value of $V_{\max }=1.9514 h^{-1}$ and $p=0.8614$. The repair function depends on the number of DSBs $(k)$ only and the attempt to repair 1 DSB is described with a probability. By specifying the repair function at $h(t)=1$, the successful attempt rate to repair 1 DSB in group $N_{4,2}$ will be $1.0324 h^{-1}$ and the unsuccessful attempt rate will be 0.1661 $h^{-1}$. It shows here the probability rate is crucial for repairing DSBs and avoid mis-repair the DSBs. Failure to repair DSBs is lethal to cells and can cause genetic defects that lead to genetic disease and secondary cancers in long term periods [46].

The modelling framework discussed here is one-step to bring advantages in clinical perspective for bystander effects phenomenon. The details on RIBE are more complex than the simplified mathematical model. The model developed is more to general process happened in bystander response and the existing experimental data to be adapted is also limited. This work can be developed further with an experimentation design in order to predict the survival bystander cells using our estimated parameter. The results of estimated parameter are obtained using the experiment of survival UVW cells after treatment with $\gamma$-irradiation and mediumtransfer from the irradiated cells. This model provides an approximation of the mechanisms of bystander cells affected by the bystander signals.

\section{Conclusion}

In this paper, a mechanistic model is proposed in order to describe the irradiation effects towards bystander cells. The modelling process is continued with data fitting and parameter estimation procedure that are able to identify the parameters value best matches with the experimental data. As shown in Figure 5(a), the model simulation by using the estimated parameters has shown a good fit with the experimental data. For further development, a close joint with radiobiologist and medical doctor should be established. Thus, the advantages of the mathematical models proposed in this paper could be explored and human quality of life can be improved.

\section{Acknowledgement}

This work was financially supported by the Universiti Teknologi Malaysia under the Fundamental Research Grant Scheme (FRGS: R.J130000.7826.4F889) and Ministry of Education Malaysia (MOE). The second author would like to thank MOE for his MyBrainSc scholarship and this paper is part of the second author's master thesis.

\section{References}

[1] Ingalls, B. Mathematical Modelling in Systems Biology: An Introduction. Canada: University of Waterloo Press. 2014. 
[2] Najafi, M., Salajegheh, A. and Rezaeyan, A. Bystander effect and second primary cancers following radiotherapy: What are its significances? J. Med. Phys. 2017. 42(1): 55.

[3] Zahrina, A. K., Norsa'adah, B., Hassan, N. B., Norazwany, Y., Norhayati, M. I., Roslan, M. H. and Wan Nazuha, W. R. Adherence to Capecitabine treatment and contributing factors among cancer patients in Malaysia. Asian Pac. J. Cancer Prev. 2014. 15(21): 9225-9232.

[4] Mothersill, C., Fernandez-Palomo, C., Fazzari, J., Smith, R., Schültke, E., Bräuer-Krisch, E., Laissue, J., Schroll, C. and Seymour, C.. Transmission of signals from rats receiving high doses of microbeam radiation to cage mates: an inter-mammal bystander effect. DoseResponse. 2014. 12(1): 72-92.

[5] Marín, A., Martín, M., Liñán, O., Alvarenga, F., López, M., Fernández, L., Büchser, D. and Cerezo, L. Bystander effects and radiotherapy. Rep. Prac. Oncol. Radiother. 2015. 20(1): 12-21.

[6] Wang, H., Yu, K. N., Hou, J., Liu, Q. and Han, W. Radiation-induced bystander effect: early process and rapid assessment. Cancer Lett. 2015. 356(1): 137-144.

[7] Najafi, M., Fardid, R., Hadadi, G. and Fardid, M. The mechanisms of radiation-induced bystander effect. J. Biomed. Phys. Eng. 2014. 4(4): 163-172.

[8] Chevalier, F., Hamdi, D. H., Saintigny, Y. and Lefaix, J. L. Proteomic overview and perspectives of the radiation-induced bystander effects. Mutat. Res. Rev. Mutat. Res. 2015. 763: $280-293$.

[9] Sprung, C. N., Ivashkevich, A., Forrester, H. B., Redon, C. E., Georgakilas, A. and Martin, O. A. Oxidative DNA damage caused by inflammation may link to stress-induced nontargeted effects. Cancer Lett. 2015. 356(1): 72-81.

[10] Verma, N. and Tiku, A. B. Significance and nature of bystander responses induced by various agents. Mutat. Res. Rev. Mutat. Res. 2017. 773: 104-121.

[11] Han, W. and Yu, K. N. Ionizing radiation, DNA double strand break and mutation. Advances in Genetics Research. 2010. 4: 197-210.

[12] Nasir, M. H. and Siam, F. M. Mini-review: recent updates on the mathematical modelling of radiation-induced bystander effects. Mal. J. Fund. Appl. Sci. 2017. 13(2): 103-108.

[13] Olobatuyi, O., de Vries, G. and Hillen, T. A reaction-diffusion model for radiation-induced bystander effects. J. Math. Biol. 2017. 75(2): 341-372.

[14] Desouky, O., Ding, N. and Zhou, G. Targeted and non-targeted effects of ionizing radiation. J. Radiat. Res. Appl. Sci. 2015. 8(2): 247-254.

[15] Widel, M. Radiation induced bystander effect: from in vitro studies to clinical application. Int. J. Med. Phys. Clin. Eng. Radiat. Oncol. 2016. 5(01): 1-17.

[16] Havaki, S., Kotsinas, A., Chronopoulos, E., Kletsas, D., Georgakilas, A. and Gorgoulis, V. G. The role of oxidative DNA damage in radiation induced bystander effect. Cancer Lett. 2015. 356(1): 43-51.

[17] Hatzi, V. I., Laskaratou, D. A., Mavragani, I. V., Nikitaki, Z., Mangelis, A., Panayiotidis, M. I., Pantelias, G. E., Terzoudi, G. I. and Georgakilas, A. G. Non-targeted radiation effects in vivo: a critical glance of the future in radiobiology. Cancer Lett. 2015. 356(1): 34-42. 
[18] Stein, G. S. and Pardee, A. B. eds., Cell Cycle and Growth Control: Biomolecular Regulation and Cancer. John Wiley \& Sons. 2004.

[19] Hall, E. J. and Giaccia, A. J. Radiobiology for the Radiologist (Vol. 6). Philadelphia: Lippincott Williams \& Wilkins. 2006.

[20] Kamran, M. Z., Ranjan, A., Kaur, N., Sur, S. and Tandon, V. Radioprotective agents: strategies and translational advances. Med. Res. Rev. 2016. 36(3): 461-493.

[21] Prise, K. M., Schettino, G., Folkard, M. and Held, K. D. New insights on cell death from radiation exposure. Lancet Oncol. 2005. 6(7): 520-528.

[22] Xia, J., Liu, L., Xue, J., Wang, Y. and Wu, L. Modeling of radiation-induced bystander effect using Monte Carlo methods. Nucl. Instrum. Methods Phys. Res. B. 2009. 267(6): 1015-1018.

[23] Sasaki, K., Wakui, K., Tsutsumi, K. and Itoh, A. A Simulation study of the radiationinduced bystander effect: modeling with stochastically defined signal reemission. Computational and Mathematical Methods in Medicine. 2012. 2012: 1-5.

[24] Kundrát, P. and Friedland, W. Non-linear response of cells to signals leads to revised characteristics of bystander effects inferred from their modelling. Int. J. Radiat. Biol. 2012. 88(10): 743-750.

[25] Lintott, R., McMahon, S., Prise, K., Addie-Lagorio, C. and Shankland, C. Using process algebra to model radiation induced bystander effects. In International Conference on Computational Methods in Systems Biology. Springer, Cham. 2014. 8859: 196-210.

[26] Hattori, Y., Yokoya, A. and Watanabe, R. Cellular Automaton-based model for radiationinduced bystander effects. BMC Syst. Biol. 2015. 9(1): 90.

[27] Siam, F. M., Grinfeld, M., Bahar, A., Rahman, H. A., Ahmad, H. and Johar, F. A mechanistic model of high dose irradiation damage. Math. Comput. Simul. 2018. 151: 156168.

[28] Liu, Y., Kobayashi, A., Maeda, T., Fu, Q., Oikawa, M., Yang, G., Konishi, T., Uchihori, Y., Hei, T. K. and Wang, Y. Target irradiation induced bystander effects between stem-like and non stem-like cancer cells. Mutat. Res. 2015. 773: 43-47.

[29] Costa, K. D., Kleinstein, S. H. and Hershberg, U. Biomedical model fitting and error analysis. Sci. Signal. 2011. 4(192): tr9.

[30] Boyd, M., Ross, S. C., Dorrens, J. and Fullerton, N. E. Radiation-induced biologic bystander effect elicited in vitro by targeted radiopharmaceuticals labeled with $\alpha-$, $\beta$-, and Auger electron-emitting radionuclides. J. Nucl. Med. 2006. 47(6): 1007-1015.

[31] Yang, H., Asaad, N. and Held, K. D. Medium-mediated intercellular communication is involved in bystander responses of X-ray-irradiated normal human fibroblasts. Oncogene. 2005. 24(12): 2096-2103.

[32] Sedelnikova, O. A., Nakamura, A., Kovalchuk, O., Koturbash, I., Mitchell, S. A., Marino, S. A., Brenner, D. J. and Bonner, W. M. DNA double-strand breaks form in bystander cells after microbeam irradiation of three-dimensional human tissue models. Cancer Res. 2007. 67(9): 4295-4302. 
[33] Dai, J., Itahana, K. and Baskar, R. Quiescence does not affect p53 and stress response by irradiation in human lung fibroblasts. Biochem. Biophys. Res. Commun. 2015. 458(1): 104-109.

[34] Powathil, G. G., Munro, A. J., Chaplain, M. A. and Swat, M. Bystander effects and their implications for clinical radiation therapy: insights from multiscale in silico experiments. J. Theor. Biol. 2016. 401: 1-14.

[35] McKibben, M. and Webster, M. D. Differential Equations with MATLAB囚: Exploration, Applications, and Theory. CRC Press. 2014.

[36] Nasir, M. H. and Siam, F. M. Simulation and sensitivity analysis on the parameter of non-targeted irradiation effects model. Jurnal Teknologi. 2019. 81(1): 133-142

[37] Lillacci, G. and Khammash, M. Parameter estimation and model selection in computational biology. PLoS Comput. Biol. 2010. 6(3): p.e1000696.

[38] Goulet, D. Modeling, simulating, and parameter fitting of biochemical kinetic experiments. SIAM Rev. 2016. 58(2): 331-353.

[39] Belchior, A., Balásházy, I., Gil, O. M., Vaz, P. and Almeida, P. Does the number of irradiated cells influence the spatial distribution of bystander effects? Dose-Response. 2014. 12(4): 525-539.

[40] Singh, S. FIR filter design using particle swarm optimization technique. International Journal of Science, Technology, Management and Research. 2017. 2(4): 54-59.

[41] Bonyadi, M. R. and Michalewicz, Z. Particle swarm optimization for single objective continuous space problem: A review. Evolutionary Computation. 2017. 25(1): 1-54.

[42] Ebbesen, S., Kiwitz, P. and Guzzella, L. A generic particle swarm optimization Matlab function. American Control Conference (ACC), 2012. IEEE. 2012. 1519-1524.

[43] Perez, R. and Behdinan, K. Particle swarm approach for structural design optimization. Computer \& Structures. 2007. 85(19): 1579-1588.

[44] Zi, Z. Mathematical Modeling and Kinetic Analysis of Cellular Signaling Pathways. Doctoral dissertation, Humboldt-Universitt zu Berlin. 2008.

[45] Schöllnberger, H., Mitchel, R.E.J., Redpath, J.L., Crawford-Brown, D.J. and Hofmann, W. Detrimental and protective bystander effects: A model approach. Radiat. Res. 2007. 168(5): 614-626.

[46] Nasir, J. Use of E18 cell model to quantify DNA strand break associated bystander effect (DSB-ABE). J. Carcinogene. Mutagene. 2017. 8(295): 27-29. 